\title{
Association of p21 with NF-YA suppresses the expression of Polo-like kinase 1 and prevents mitotic death in response to DNA damage
}

\author{
Y-C Lin ${ }^{1}$, Y-N Chen ${ }^{1}$, K-F Lin ${ }^{1}$, F-F Wang ${ }^{1}$, T-Y Chou ${ }^{\star 1,1,3}$ and M-Y Chen ${ }^{\star, 1,4}$
}

Polo-like kinase 1 (PLK1) is an important mitotic kinase and its expression is tightly regulated in the cell cycle and in the DNA damage response. PLK1 expression is previously shown to be suppressed by p53 and/or p21. Here, we demonstrate that the CCAAT box in the PLK1 promoter is pivotal for p53/p21-mediated PLK1 repression. Chromatin immunoprecipitation showed that cyclin-dependent kinase 2 (CDK2) associated with the CCAAT box-containing region of PLK1 promoter in unstressed cells, whereas adriamycin (ADR) induced the recruitment of p21 with a concomitant reduction in the occupancy of CDK2 in this region. Expression of p21 inhibited the interaction between CDK2 and the nuclear factor YA (NF-YA) subunit of the CCAAT box-binding transcription factor NF-Y. A mutant p21 that is defective in CDK2 binding was unable to disrupt the CDK2-NF-YA interaction or suppress PLK1 transcription. Co-immunoprecipitation experiments demonstrated the interaction between NF-YA and p21, and in vitro assays showed that p21 could directly bind to NF-YA. Knockdown of NF-YA decreased the amount of PLK1 promoterassociated p21 and abolished p21-mediated PLK1 repression in cells treated with ADR. Depletion of NF-YA diminished the p53regulated transcriptional activation and suppressed the p53-mediated protection from mitotic death after DNA damage, and these effects of NF-YA deletion were alleviated by PLK1 depletion. Our findings have uncovered a novel p21/NF-YA/PLK1 axis critical for maintaining the checkpoint function of p53 to prevent mitotic death in the DNA damage-induced response.

Cell Death and Disease (2014) 5, e987; doi:10.1038/cddis.2013.527; published online 9 January 2014

Subject Category: Cancer

Polo-like kinase 1 (PLK1) is a Ser/Thr kinase that has important roles in multiple phases of mitosis; its function is involved in centrosome maturation, mitotic entry, anaphase progression and mitotic exit. ${ }^{1-3}$ PLK1 is tightly controlled in its abundance and activity during cell cycle progression., ${ }^{1,4}$ Protein and mRNA levels of PLK1 remain low throughout G1 and S phases, start to increase in the G2 phase and peak during mitosis; the concordant changes in transcript and protein levels suggest that PLK1 expression is primarily controlled transcriptionally in these phases of cell cycle., The cell cycle-dependent element (CDE)/cell cycle gene homology region (CHR) in the PLK1 promoter represents a key transcriptional repression element; although the responsible trans-acting factors remain elusive, mutations in this bipartite cis element hinder the cell cycle-specific regulation of $P L K 1{ }^{4,5}$ Upon the mitotic exit, PLK1 is regulated at the protein level by anaphase-promoting complex/cyclosome-mediated ubiquitination, which destines PLK1 for proteosomal degradation. ${ }^{6}$

The expression of PLK1 is also regulated under genotoxic stress conditions. ${ }^{7,8}$ Both the expression and activity of PLK1 are downregulated upon the activation of the DNA damage-induced G2/M checkpoint, which prevents mitotic entry in p53-dependent and -independent manners. ${ }^{7,9}$ The transcriptional repression of $P L K 1$ induced by DNA damage can occur through the activation of $\mathrm{G} 2 / \mathrm{M}$ checkpoint kinases ATM and ATR, and is dependent on functional p53 and/or p21. ${ }^{10-12}$ The repressive effect of $\mathrm{p} 53$ on PLK1 expression appears to be CDE/CHR independent and involves the binding of p53 to a response element (p53RE2) 800-bp upstream of the $\mathrm{CDE} / \mathrm{CHR}$ element and the recruitment of histone deacetylase to the vicinity of p53RE2. ${ }^{13}$ Deletion studies suggest that the CDE/CHR element is important in p21-mediated repression of the $P L K 1,{ }^{14}$ but the underlying mechanism has not been well elucidated. The PLK1 promoter also contains one single CCAAT box, which is essential for the promoter activity; ${ }^{15}$ the role of this element in stressresponsive PLK1 regulation has not been explored yet.

Nuclear factor $Y(N F-Y)$ is a CCAAT box-binding transcription factor composed of three different subunits: $Y A, Y B$ and YC. The association between NF-YB and NF-YC provides a docking site for NF-YA, and NF-YA is responsible for sequence-specific DNA binding. ${ }^{16,17} \mathrm{NF}-\mathrm{Y}$-binding loci in the

\footnotetext{
${ }^{1}$ Institute of Biochemistry and Molecular Biology, National Yang-Ming University, Taipei, Taiwan; ${ }^{2}$ Institute of Clinical Medicine, National Yang-Ming University, Taipei, Taiwan; ${ }^{3}$ Department of Pathology and Laboratory Medicine, Taipei Veterans General Hospital, Taipei, Taiwan and ${ }^{4}$ Cancer Research Center and Genome Research Center, National Yang-Ming University, Taipei, Taiwan

${ }^{*}$ Corresponding author: T-Y Chou, Department of Pathology and Laboratory Medicine, Taipei Veterans General Hospital, 201, Section 2, Shih-Pai Road, Taipei 11217, Taiwan. Tel: +886 22875 7080; Fax: +886 22875 7056; E-mail: tychou@vghtpe.gov.tw or M-Y Chen, Institute of Biochemistry and Molecular Biology, National Yang-Ming University, No. 155, Section 2, Li-Nong Street, Beitou District, Taipei 11221 , Taiwan. Tel: +886 22826 7269; Fax: +886 22826 4843; E-mail: meychen@ym.edu.tw

Keywords: PLK1; NF-YA; p21; p53; mitotic death

Abbreviations: ADR, adriamycin; CDE, cell cycle-dependent element; CDK2, cyclin-dependent kinase 2; ChIP, chromatin immunoprecipitation; Co-IP, coimmunoprecipitation; CHR, cell cycle gene homology region; PCR, polymerase chain reaction; PLK1, Polo-like kinase 1; RT, reverse transcription

Received 04.9.13; revised 24.11.13; accepted 26.11.13; Edited by D Aberdam
} 
genome can be decorated with either positive or negative methyl-histone marks, indicating that NF-Y may serve as either an activator or a repressor in the transcriptional regulation of target genes. ${ }^{18} \mathrm{NF}-\mathrm{Y}$ may cooperate with transcriptional cofactors or neighboring transcription factors in modulating gene transcription. For example, NF-Y can either repress or activate the promoter of the von Willebrand factor (VWF) gene, depending on its association with histone deacetylase 1 or p300/CBP-associated factor. ${ }^{19,20}$ In regulating the $\gamma$-globulin gene, NF-Y may activate or repress $\gamma$-globulin expression by recruiting the transcriptional activator GATA-2 or the repressors GATA-1 and COUP-TFII, respectively, to the neighboring DNA motifs. ${ }^{21}$ Moreover, the transcriptional effect of NF-Y can be modulated by post-translational modifications. Cyclin-dependent kinase 2 (CDK2)-mediated phosphorylation of NF-YA positively regulates NF-Y-mediated activation of CCAAT box-containing genes such as CDK1, E2F1, CCNA2 and $C D C 25 C,{ }^{22}$ whereas a phosphorylation-deficient mutant NF-YA represses cell cycle-regulated genes. ${ }^{23}$ Whether any of the above-mentioned NF-Y-dependent regulatory mechanisms participates in transcriptional regulation of $P L K 1$ is still unknown.

We have reported that $\mathrm{p} 21$-mediated suppression of PLK1 is responsible for the p53-mediated inhibition of mitotic catastrophe in cells with DNA damage. ${ }^{11}$ Here, we provide evidence showing that regulation of PLK1 expression may be fine-tuned through dynamic interactions among NF-YA, p21 and CDK2 on the PLK1 promoter. We demonstrate that while CDK2 resides in the CCAAT box-containing region of the PLK1 promoter in unstressed cells, p21 binds to CDK2 and frees it from interacting with NF-YA; NF-YA, in turn, binds and anchors p21 to the PLK1 promoter and the two regulatory factors cooperate to inhibit PLK1 transcription in cells with DNA damage. Significantly, the NF-YA-mediated suppression of PLK1 is necessary to prevent adriamycin (ADR)-induced mitotic death, indicating that this novel mechanism for $P L K 1$ regulation underlies the cell fate determination after DNA damage.

\section{Results}

The CCAAT box in the PLK1 promoter is required for p53/p21-dependent transcriptional repression of PLK1 in response to DNA damage. We have previously shown in H1299 cells stably transfected with a temperature-sensitive p53 mutant (tsp53) that the induction of functional p53 decreases PLK1 protein levels in a p21-dependent manner. ${ }^{11}$ In this study, we found that ADR treatment could increase p53 expression in HCT116 cells with or without p21 deletion, whereas levels of PLK1 protein and transcript were only decreased in the wild-type but not in the p21-/- cells (Figure 1a). These data confirmed the requirement of p21 in suppressing PLK1 expression in response to the DNAdamaging reagent, and suggested a regulatory mechanism at the transcriptional level. We constructed a reporter plasmid pGL3-Luc-PLK1, which contains the $-1717 /+58$-bp region of the PLK1 promoter, and tested its promoter activity in the isogenic HCT116 cell system. Results of luciferase assays showed that ADR treatment inhibited the PLK1 promoter activity in the wild-type but not in the $\mathrm{p} 21^{-/-}$or $\mathrm{p} 53^{-/-}$cells
(Figure 1b), indicating that the DNA damage-induced transcriptional repression is dependent on the p53/p21 pathway. Introducing a p21-expressing plasmid into $\mathrm{p} 21^{-1-}$ or $\mathrm{p} 53^{-1-}$ cells restored the ADR-induced PLK1 promoter inhibition, demonstrating that p21 is sufficient for this transcriptional repression.

To investigate the importance of the CCAAT box in the PLK1 promoter for p53/p21-mediated repression, a pGL3Luc-PLK1 variant carrying a mutated CCAAT box (mCCAAT; CCAAT to CAGCT) was prepared and compared with the wild-type reporter construct (wtCCAAT) in the previously established tsp53- and neo-H1299 (vector control) stable clones. ${ }^{11}$ Transfected cells were subjected to the temperature shift (for the induction of functional p53 in tsp53-H1299 cells) and treated by ADR to induce DNA damage, and their lysates were assayed for luciferase activities (Figure 1c). The activity of the wtCCAAT promoter in tsp53-H1299 cells was significantly lower than that in the neo-H1299 control clone, and this repressive effect of p53 could be alleviated by p21 knockdown; however, the mCCAAT promoter was unresponsive to p53 activation. Consistent with the above results, p21 overexpression in the p53-null $\mathrm{H} 1299$ background was sufficient to inhibit the activity of the wtCCAAT but not the mCCAAT promoter (Figure 1d). In H1299 and neo-H1299 cells, where p53 and p21 were not expressed, the mutation of CCAAT did not affect the PLK1 promoter activity (Figures $1 c$ and $d$ ). Collectively, our results suggest that the CCAAT box is essential for the DNA damage-induced p53/p21-dependent PLK1 repression.

p21 is recruited to a PLK1 promoter region containing the CCAAT box in response to DNA damage. To explore the mechanism by which $\mathrm{p} 21$ regulates PLK1 in cells with DNA damage, we tested if p21 can be recruited to the promoter following ADR treatment. Chromatin immunoprecipitation (ChIP) assays were performed using specific antibodies and primer sets to amplify regions of the PLK1 promoter from precipitated DNA (Figure 2a); region A was used as a control whereas region $B$ is the region containing the CCAAT box $(-39 /-35 \mathrm{bp}) .{ }^{14}$ ADR treatment induced the recruitment of p21 specifically to the CCAAT boxcontaining region in the PLK1 promoter in wild-type but not $\mathrm{p} 21^{-/-}$or $\mathrm{p} 53^{-/-}$HCT116 cells (Figure $2 \mathrm{~b}$ ). ChIP assays using an antibody against NF-YA, that is, the DNA-binding subunit of the CCAAT box-binding NF-Y, showed that NF-YA associated with the CCAAT box-containing PLK1 promoter region in wild-type, $\mathrm{p} 21^{-/-}$or $\mathrm{p} 53^{-/-}$HCT116 cells, either with or without ADR treatment (Figure 2b). CDK2, a kinase known to bind and phosphorylate NF-YA, ${ }^{22}$ also associated with this promoter region; intriguingly, the association of CDK2 with promoter was markedly reduced following ADR treatment in wild-type HCT116 cells, but less evident in $\mathrm{p} 21^{-/-}$or $\mathrm{p} 53^{-/-}$cells. Similar patterns of promoter occupancy were observed in the tsp53-H1299 cells that were induced to express functional p53: NF-YA was present on the PLK1 promoter in the CCAAT box-containing region whether cells were treated with ADR or not, whereas p21 was recruited to this promoter region only after ADR treatment; CDK2 associated with the promoter in untreated cells but not in cells with induced DNA damage (Figure 2c). 

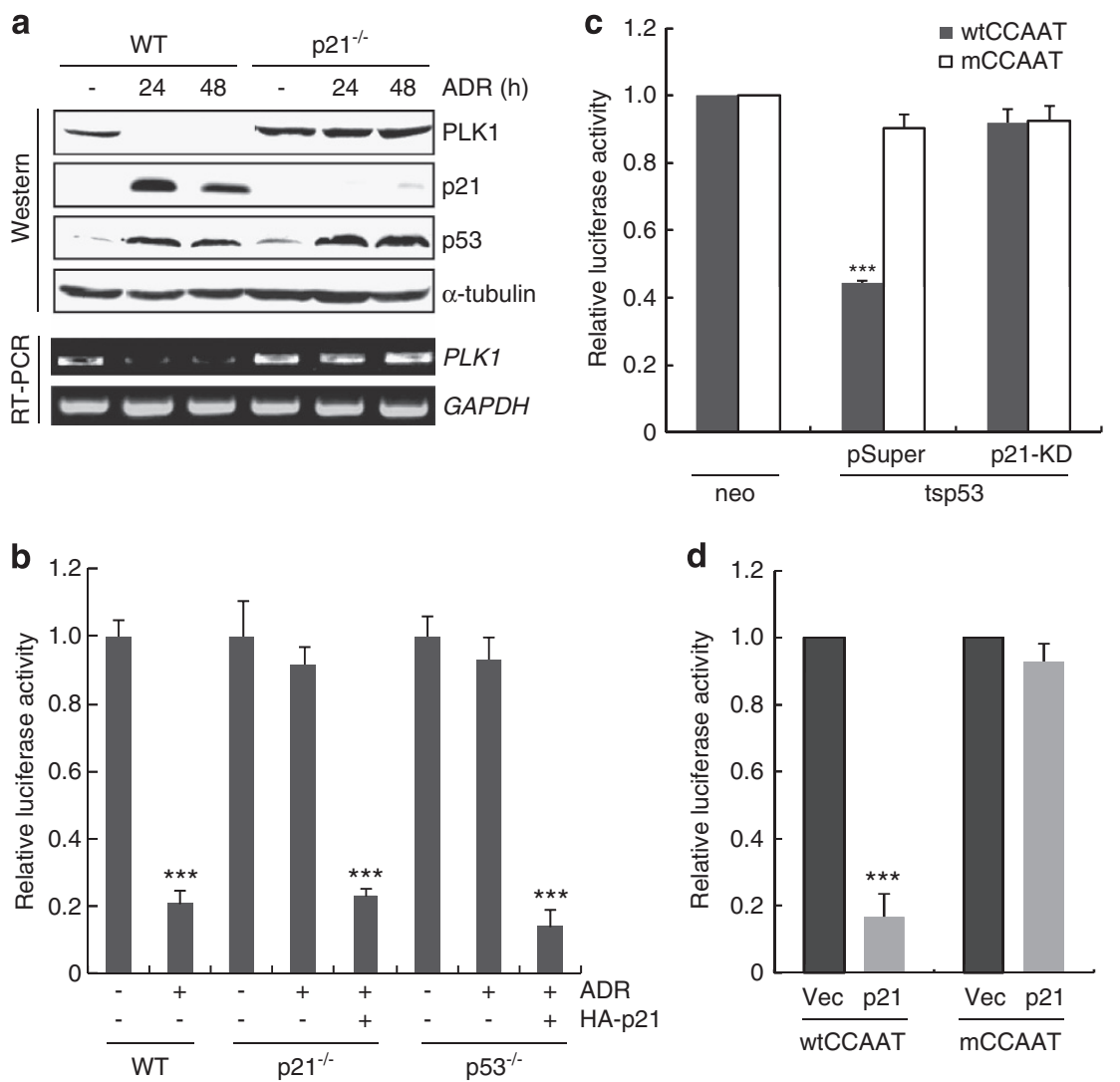

Figure 1 The CCAAT box in PLK1 promoter is pivotal for p21-mediated PLK1 repression in response to ADR treatment. (a) Isogenic p21 ${ }^{+/+}\left(\right.$WT) and p21 ${ }^{-1-}$ HCT116 cells were treated with ADR for $12 \mathrm{~h}$ and subsequently cultured in the ADR-free medium for 24 or $48 \mathrm{~h}$; -, no ADR treatment. Lysates and RNA samples were analyzed by immunoblotting and RT-PCR, respectively. (Note: an ADR concentration of $400 \mu \mathrm{g} / \mathrm{ml}$, which triggered apoptosis in $>40 \%$ of the cells was used throughout this study; treating cells at lower ADR concentrations such as $50 \mu \mathrm{g} / \mathrm{ml}$ resulted predominantly in cell cycle arrest, and PLK1 repression was not detectable). (b) Isogenic p21 ${ }^{+/+} \mathrm{p} 53^{+/+}(\mathrm{WT})$, $\mathrm{p} 21^{-1-}$ or $\mathrm{p} 53^{-1-}$ HCT116 cells were transfected with a firefly luciferase reporter plasmid containing the PLK1 promoter (pGL3-Luc-PLK1) and a TK-Renilla luciferase expression vector (pRL-TK) with or without an HA-p21-expressing plasmid, and treated with ADR as in (a). Cell lysates were prepared $24 \mathrm{~h}$ after the release from ADR treatment and subjected to dual luciferase activity assays. (c) The control vector-transfected (neo) or the tsp53-expressing stable H1299 clones were transfected with luciferase reporter constructs and the pSuper control vector or the p21-knockdown shRNA-expressing plasmid (p21-KD). Cells were cultured at $38^{\circ} \mathrm{C}$ for $24 \mathrm{~h}$, shifted to $32^{\circ} \mathrm{C}$ and treated with $A D R$ for $12 \mathrm{~h}$, and transferred to a drug-free medium at $32^{\circ} \mathrm{C}$ for $24 \mathrm{~h}$ before the luciferase activity was assayed. (d) H1299 cells were transfected with pGL3Luc-PLK1 (wtCCAAT) or the CCAAT box-mutated construct pGL3-Luc-mutPLK1 (mCCAAT) and pRL-TK in the presence or absence of a p21-expressing plasmid; the luciferase activity was assayed at $24 \mathrm{~h}$ after transfection. (b-d) Normalized luciferase activities were compared with that of the control sample (which was set as 1 ) in each set of experiments. Bars represent mean \pm S.D. from three independent experiments ${ }^{* * *} P<0.001$, Student's $t$-test)

The occupancy of the $C D C 25 A$ promoter was also examined as a control; ADR treatment promoted the recruitment of p21 to the $-222 /-58$-bp region that contains two CCAAT boxes ${ }^{24}$ which was accompanied by a reduction in CDK2 association to the same region. The reciprocal changes in the amounts of promoter-associated p21 and CDK2 in response to DNA damage suggest that p21 may regulate transcription of those target genes with CCAAT box-containing promoters by replacing CDK2 in binding to CCAAT boxbound NF-Y.

Expression of p21 disrupts the interaction between CDK2 and NF-YA. It is known that CDK2 can form a complex with NF-YA. ${ }^{23}$ By expressing a fixed amount of NF-YA with increasing dosage of p21 in H1299 cells, we tested if p21 could affect the formation of the CDK2/NF-YA complex. Co-immunoprecipitation (co-IP) experiments showed that increasing p21 expression resulted in decreasing amounts of NF-YA detected in the CDK2 immunoprecipitates (Figure 3a).
To confirm this effect of p21, we used the tsp53-H1299 cell system; cells were induced to express functional p53 and treated with ADR to activate the p53/p21 pathway, and cell lysates were subjected to CDK2 immunoprecipitation combined with immunoblotting analysis to detect the association between NF-YA and CDK2. In vector-transfected tsp53H1299 cells with induced p21 expression, the CDK2/NF-YA complex could not be detected. In the p53-null neo-H1299 control cells and the tsp53-H1299 cells with p21 knockdown (p21-KD), where p21 expression could not be induced, NF-YA was co-immunoprecipitated with CDK2 (Figure 3b). These results support a role of p21 in negatively regulating the association between CDK2 and NF-YA

As p21 can bind to CDK2, ${ }^{25}$ we investigated if the CDK2binding ability is required for the above-described p21 effect. A CDK2-binding-defective p21 mutant, p21-PRG, was tested. $^{26}$ In co-IP experiments, unlike wild-type p21, p21$\mathrm{PRG}$ was unable to reduce the association between CDK2 and NF-YA in the cellular context (Figure $3 \mathrm{c}$ ). ChIP assays 


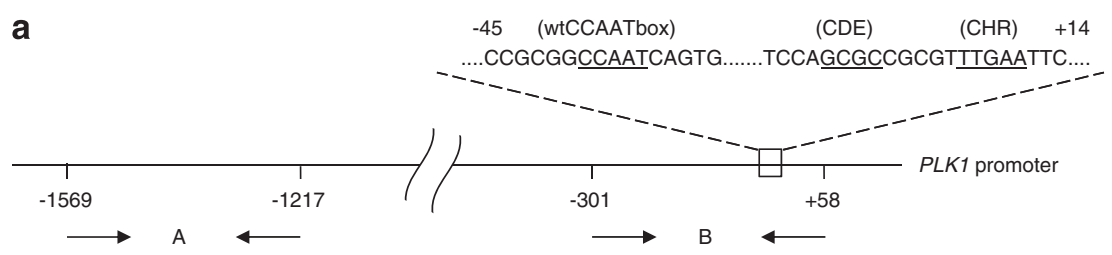

b

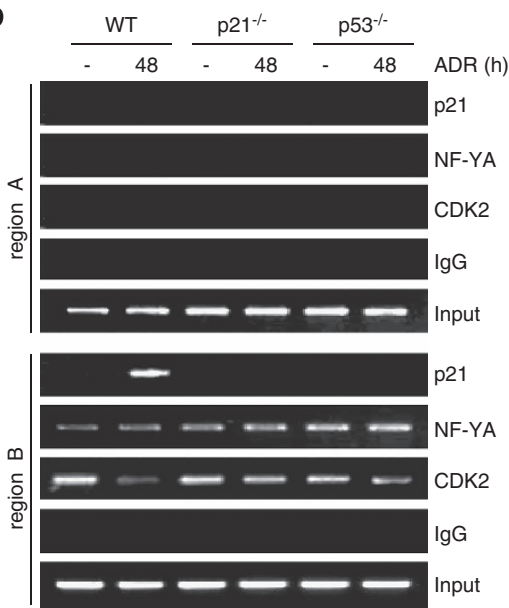

C

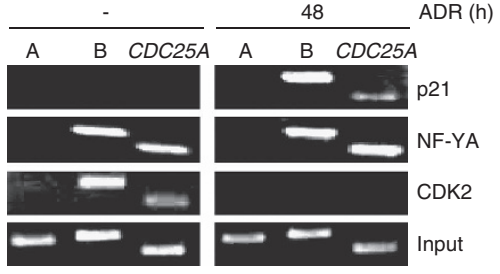

Figure 2 p21 is recruited to the CCAAT box-containing region of PLK1 promoter in response to ADR treatment. (a) A schematic representation of the PLK1 promoter. The regions ( $\mathrm{A}$ and $\mathrm{B}$ ) amplified in ChIP assays are indicated. Nucleotide positions are numbered relative to the translational start site $(+1)$. The locations of wtCCAAT box, CDE and $\mathrm{CHR}$ are indicated and their exact sequences are underlined. ( $\mathbf{b}$ and $\mathbf{c})$ Recruitment of regulatory proteins to $P L K 1$ promoter regions was assessed by $\mathrm{ChIP}$ assays using antibodies against p21, CDK2 or NF-YA. The - 1569/ - 1217-bp region A as a control and the $-301 /+58$-bp region B that contains the CCAAT box were amplified by PCR using specific

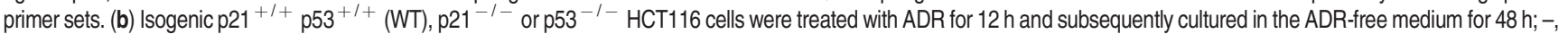
no ADR treatment. (c) The tsp53-H1299 cells were cultured at $38^{\circ} \mathrm{C}$ for $24 \mathrm{~h}$, shifted to $32^{\circ} \mathrm{C}$ and treated with $\mathrm{ADR}$ for $12 \mathrm{~h}$, and transferred to drug-free medium at $32^{\circ} \mathrm{C}$ for $48 \mathrm{~h}$ before samples were prepared for ChIP; -, no ADR treatment. The p21 response element ( $-222 /-58 \mathrm{bp})$ in the CDC25A promoter was also amplified as a control

showed that the amount of PLK1 promoter-associated CDK2 was significantly reduced when wild-type p21, but not p21-PRG, was overexpressed (Figure 3d). Compared with the wild-type p21, p21-PRG was less effective in suppressing the PLK1 promoter activity (Figure $3 e$ ) and in lowering the PLK1 mRNA levels (Figure 3f). Together, these results suggest that the CDK2-binding ability is required for p21 to interfere with the formation of the CDK2/NF-YA complex and to suppress PLK1 expression.

NF-YA binds to p21 directly and anchors p21 to the CCAAT box-containing promoter region for PLK1 repression. As both $\mathrm{p} 21$ and NF-YA were detected in the CCAAT box-containing region of the PLK1 promoter, the possibility that p21 binds to NF-YA was explored. Immunoprecipitation and immunoblotting on lysates from 293T cells co-expressing HA-tagged p21 and Flag-tagged NF-YA revealed that $\mathrm{p} 21$ could interact with NF-YA within cells (Figure 4a). Recombinant GST-tagged p21 purified from E. coli could associate with in vitro translated ${ }^{35} \mathrm{~S}$-labeled NF-YA (Figure 4b). Further in vitro binding analysis using GST-tagged NF-YA and His-tagged p21 purified from $E$. coli demonstrated that NF-YA could directly interact with p21 (Figure 4c). We also tested p21-PRG and found it able to bind to NF-YA in the in vitro GST pull-down assay (Figure 4c). However, co-IP showed that, unlike the wildtype p21, p21-PRG failed to interact with NF-YA (Figure 4d), supporting that the CDK2-binding ability is required for p21 to interact with NF-YA in the cellular context.
The role of NF-YA in anchoring p21 to the PLK1 promoter was next examined. In Flag-p21-expressing H1299 cells, ChIP analysis demonstrated that NF-YA knockdown caused a significant reduction of the amount of p21 bound to the CCAAT box-containing region of PLK1 promoter (Figure 4e). In contrast, compared with the control, overexpression of wild-type NF-YA but not a mutant NF-YA (m29) impaired in DNA binding ${ }^{27}$ increased the amount of promoter-associated p21 (Figure 4f). Similarly, expression of NF-YA in tsp53H1299 cells further increased the amount of promoterassociated p21 following p53 induction (Figure $4 \mathrm{~g}$ ). These results suggest that $\mathrm{p} 21$ associates with the $P L K 1$ promoter through its interaction with the CCAAT box-bound NF-YA.

NF-YA has a pivotal role in p53/DNA damage-induced $P L K 1$ repression. The role of NF-YA in p53-mediated PLK1 suppression during DNA damage response was also investigated. The tsp53-H1299 cells were co-transfected with the pGL3-Luc-PLK1 reporter construct together with a control vector (shVec) or a plasmid-expressing NF-YAtargeting shRNAs; lysates for the luciferase assay were prepared from transfected cells after temperature-induced expression of functional p53 and ADR treatment. Comparing vector-transfected p53-null neo-H1299 control and tsp53H1299 cells, p53 induction/DNA damage resulted in suppression of the PLK1 promoter activity and this suppression was alleviated by the silencing of NF-YA expression (Figure 5a). Reverse transcription (RT)-PCR analysis demonstrated that NF-YA knockdown decreased the 
a

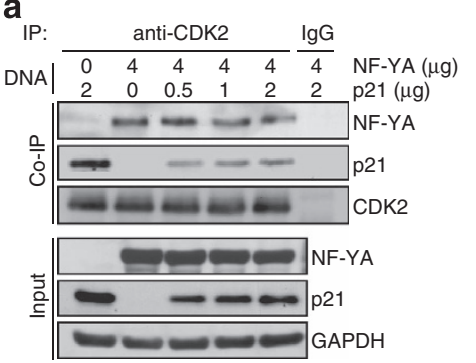

b

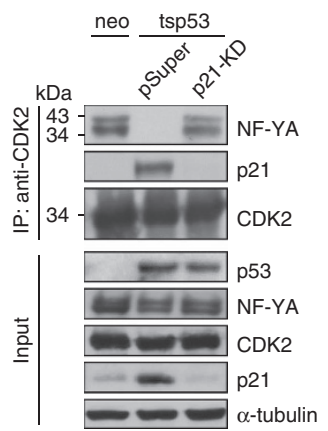

C

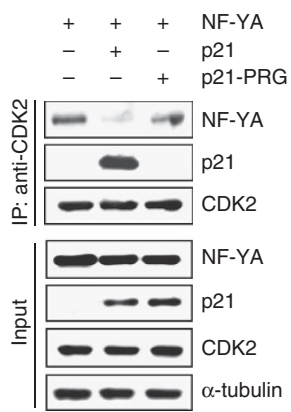

d

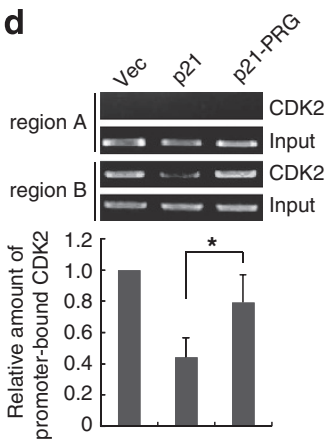

e

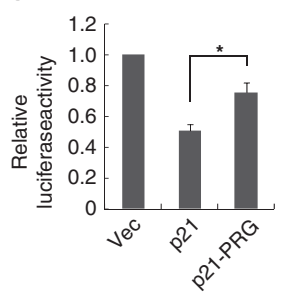

f

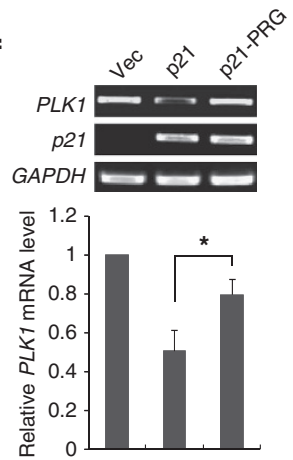

Figure 3 Expression of $\mathrm{p} 21$ disrupts the interaction between NF-YA and CDK2. (a-c) Co-IP assays with anti-CDK2 antibodies. Proteins in the input lysates or immunoprecipitates were analyzed by immunoblotting using specific antibodies as indicated. (a) H1299 cells were co-transfected with the indicated amounts of FlagNF-YA- and HA-p21-expressing plasmids. Lysates were prepared $24 \mathrm{~h}$ after transfection and immunoprecipitated with anti-CDK2 antibodies or with $\operatorname{lgG}$ as a control. (b) Control (neo) and tsp53-1299 cells without (pSuper) or with p21 depletion (p21-KD) were treated with ADR for $12 \mathrm{~h}$ and transferred to a drug-free medium at $32{ }^{\circ} \mathrm{C}$ for $24 \mathrm{~h}$ before lysates were prepared for immunoprecipitation. (c) Lysates were prepared from H1299 cells overexpressing NF-YA and Flag-tagged p21 or p21-PRG, and subjected to immunoprecipitation with anti-CDK2 antibodies. (d-f) $\mathrm{H} 1299$ cells transfected with the control vector or a plasmid-overexpressing Flag-tagged p21 or p21-PRG were analyzed. Bars represent the mean \pm S.D. from three experiments $\left({ }^{*} P<0.05\right.$, Student's $t$-test). (d) ChIP assays using anti-CDK2 antibodies. The precipitated chromosomal DNA was amplified by PCR using primer sets for regions $A$ and $B$ shown in Figure 2a. Relative levels of promoter-bound CDK2 were quantified by normalizing the amount of PCR product of region $B$ from ChIP precipitates to that from the input samples, and the results are shown as relative values compared with the vector-transfected control (Vec). (e) Cells were co-transfected with pGL3-Luc-PLK1 and pRL-TK for $24 \mathrm{~h}$ and lysates were prepared for luciferase assays. Normalized luciferase activities were compared with that of the vector control. (f) RT-PCR analysis. The amount of PLK1 transcript relative to that of GAPDH was determined for each sample and normalized to the value obtained from the vector control sample (Vec), which was set as 1

suppressive effect of $\mathrm{p} 53$ induction/ADR treatment on PLK1 mRNA expression in tsp53-H1299 cells, indicating that NF-YA is important for the DNA damage-induced PLK1 repression (Figure 5b). To investigate whether the

DNA-binding activity of NF-YA is required for the p21-mediated PLK1 inhibition, tsp53-H1299 cells were transfected to express wild-type or mutant NF-YA that are impaired in DNA binding (m28 or m29); ${ }^{27}$ transfected cells were induced by the temperature shift to activate the p53-p21 axis, and cell lysates were prepared for luciferase assays. The results showed that both NF-YA mutants did not suppress the PLK1 promoter activity but the wild-type NF-YA did (Figure 5c), indicating that the DNA-binding activity of NF-YA is required for repressing PLK1. Together, these results suggest that the promoter-bound NF-YA is essential for the p53/p21-dependent inhibition of PLK1 expression in the DNA damage response.

NF-YA-mediated suppression of PLK1 expression is important for the p53 checkpoint function and prevention of mitotic death following DNA damage. We have previously shown that $\mathrm{p} 21$-mediated suppression of PLK1 is necessary for the checkpoint function of p53. ${ }^{11}$ Given the above findings suggesting that NF-YA is required for p53-mediated repression of $P L K 1$, we examined the functional significance of NF-YA in the p53-mediated stress response. In HCT116 cells transfected with a reporter construct containing the p53 response element, luciferase assays showed that NF-YA silencing, similar to p21 depletion, decreased the ADR-induced p53 transcriptional activity (Figure 6a). In tsp53-H1299 cells, RT-PCR analysis demonstrated that NF-YA silencing significantly reduced ADR-induced expression of p53 target genes p21 and MDM2 (Figure 6b). In NF-YA knocked-down tsp53-H1299 cells, PLK1 silencing abrogated the suppressive effect of NF-YA depletion on p53 transcriptional activity (Figure 6c), suggesting that the increased PLK1 expression resulting from NF-YA silencing may underlie the inhibition of p53 activity.

We have previously shown that p53 protects cells from ADR-induced mitotic death. ${ }^{28}$ By examining the formation of multinucleated cells and the increase in subG1 DNA content to assess mitotic death, we investigated the functional significance of NF-YA in the mitotic checkpoint function of p53. Compared with the control, HCT116 cells depleted of NF-YA and $\mathrm{p} 21^{-/-}$cells similarly displayed a significant increase in the formation of multinucleated cells following exposure to ADR (Figure 6d), suggesting that both p21 and NF-YA participate in protecting cells from mitotic death. Analysis of the subG1 DNA content in ADR-treated tsp53H1299 cells revealed that NF-YA silencing significantly increased the subG1 cell population; this effect was diminished by simultaneous knockdown of PLK1, suggesting that the effect of NF-YA depletion on mitotic death resulted from increased PLK1 expression (Figure 6e). Colony formation assays verified that depletion of NF-YA decreased the survival of ADR-treated cells. The clonogenic activity of NF-YA-depleted cells was substantially reduced relative to the vector control cells, and the depletion of PLK1 in these cells partially restored the colony formation activity (Figure 6f). Collectively, our results suggest that by repressing PLK1 expression, NF-YA has an important role in the p53 checkpoint function that helps to prevent DNA damageinduced mitotic death. 
a

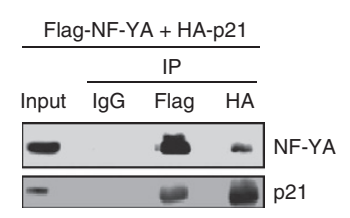

b
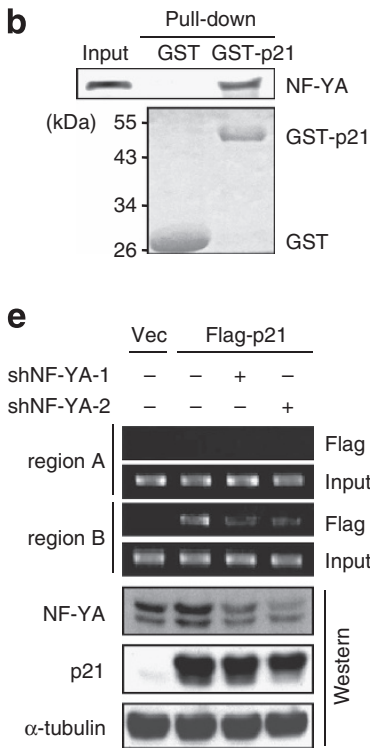

C

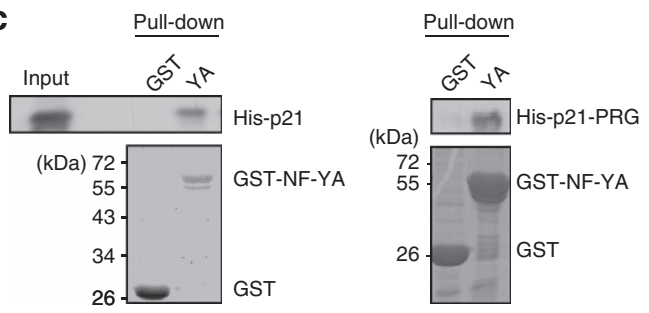

d Input: p21 p21-PRG p21 p21-PRG

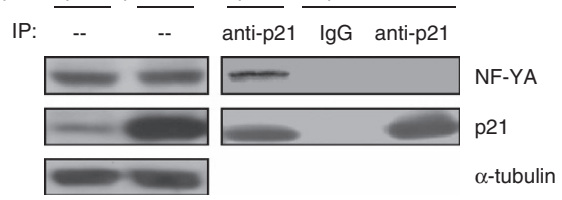

f

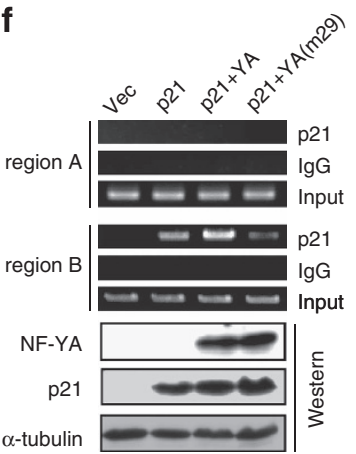

g

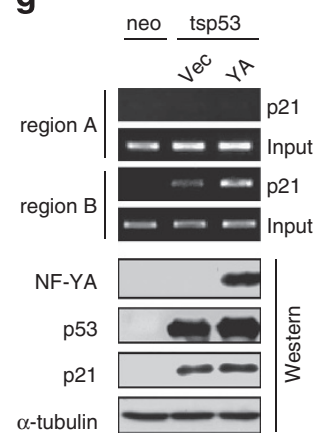

Figure 4 NF-YA binds to p21 directly and anchors p21 to the CCAAT box-containing region in the PLK1 promoter. (a) Co-IP assays. Plasmids expressing Flag-NF-YA and HA-p21 were co-transfected into 293T cells. Lysates were immunoprecipitated with anti-Flag or anti-HA antibodies, and the amounts of NF-YA and p21 in the precipitates were determined by immunoblotting. (b and $\mathbf{c}$ ) In vitro binding assays. Proteins bound to glutathione-sepharose beads were resolved by SDS-PAGE and detected by immunoblotting for the pulled-down proteins or Coomassie blue staining for GST-tagged proteins. (b) Recombinant GST-tagged p21 from E. coli was immobilized on glutathione-sepharose beads and incubated with ${ }^{35}$ S-labeled NF-YA prepared by in vitro translation. (c) Recombinant GST-tagged NF-YA from E. coli was immobilized on glutathione-sepharose beads and incubated with purified His-tagged p21 or p21-PRG. (d) Co-IP assays. Lysates were prepared from H1299 cells overexpressing NF-YA and Flag-tagged p21 or p21-PRG, and subjected to immunoprecipitation with anti-p21 antibodies. Proteins in the input lysates or precipitates were analyzed by immunoblotting using specific antibodies. (e-g) ChIP assays. Immunoprecipitation were performed using anti-Flag or anti-p21 antibodies. DNA in precipitates was amplified using primer sets for regions A and B shown in Figure 2a. Amounts of promoter-associated Flag-p21 or p21 were normalized to the individual inputs and compared with the value of the vector control sample (which was set as 1). Expression levels of NF-YA and p21 in samples were also analyzed by immunoblotting. (e) H1299 cells transfected with the control vector (Vec) or the Flag-p21-expressing plasmid in the absence or presence of NF-YA-specific shRNA expression were analyzed at $24 \mathrm{~h}$ after transfection. (f) $\mathrm{H} 1299 \mathrm{cellls}$ transfected with a Flag-p21-expressing plasmid alone or in combination with wild-type or mutant NF-YA (m29) were analyzed at $24 \mathrm{~h}$ after transfection. (g) The tsp53-H1299 cells transfected with a plasmid-expressing NF-YA were cultured at $32{ }^{\circ} \mathrm{C}$ for $24 \mathrm{~h}$ (for the induction of functional p53) and analyzed

\section{Discussion}

We have uncovered that beyond its well-known role as a p53 downstream checkpoint component that binds CDK2 and halts cell cycle progression, p21 can directly bind to NF-YA on the PLK1 promoter and actively suppress $P L K 1$ transcription to inhibit mitotic entry and prevent mitotic catastrophe. We propose a model of NF-Y-mediated transcriptional regulation of PLK1 expression (Figure 7). In unstressed cells, NF-YA is associated with and phosphorylated by CDK2, which facilitates NF-Y binding to the CCAAT box and activates transcription $^{22}$ presumably by recruiting coactivators with the histone acetyltransferase activity to the $P L K 1$ promoter region to acquire an active chromatin environment; ${ }^{20}$ the cell cycle-dependent regulation of CDK2 may help to ensure the normal progression of cell cycle in this scenario. Upon DNA damage, the activation of p53 leads to increased p21 levels, which causes the replacement of CDK2 by $p 21$ in interacting with the CCAAT box-bound NF-YA, turning the CCAAT boxassociated regulatory complex into a repressive one; presumably through recruitment of corepressors with the histone deacetylase activity to remove the acetylation of histones, ${ }^{20}$ the p21/NF-Y complex causes transcriptional repression of $P L K 1$ and thereby prevents mitotic catastrophe. Notably, we have also observed the replacement of CDK2 by p21 in associating with the CCAAT box in the promoter of another p21-repressible gene, $C D C 25 A$, after ADR treatment. ${ }^{14,24}$ This finding suggests that the dynamic interplay among CDK2, p21 and NF-YA might be a commonly adopted mechanism in regulating CCAAT box-containing promoters in response to DNA damage.

Our results show that p21-PRG, a p21 mutant defective in CDK2 binding, retains the ability to directly interact with NFYA in vitro, suggesting that the CDK-interacting and NF-YAinteracting regions of p21 are independent. In the cellular context, however, p21-PRG does not bind CDK2 or interact 
a
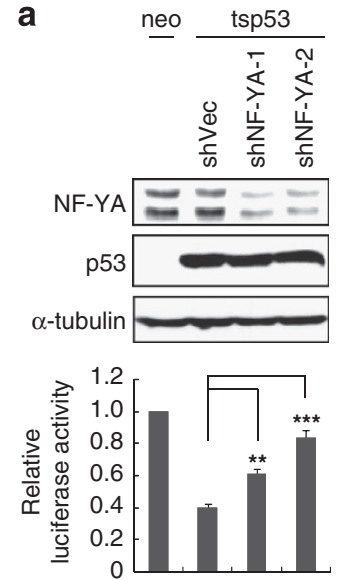

b
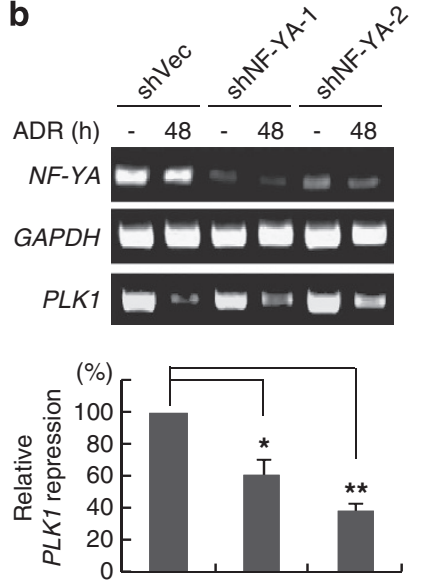

C

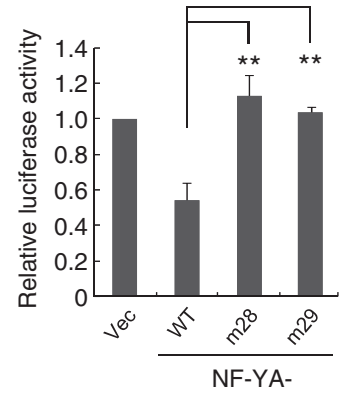

Figure 5 NF-YA has an important role in p53/DNA damage-induced PLK1 repression. (a) NF-YA-depleted (shNF-YA-1, shNF-YA-2) and vector control (shVec) tsp53H1299 cells were subjected to luciferase reporter assays after being co-transfected with pGL3-Luc-PLK1 and pRL-TK and treated with ADR as described in Figures 1c. (b) NF-YA-depleted (shNF-YA-1, shNF-YA-2) and vector control (shVec) tsp53-H1299 cells were treated with ADR and assayed for the expression of the indicated genes by RT-PCR. Expression of $P L K 1$ at $48 \mathrm{~h}$ after ADR release was normalized to the GAPDH transcript level, and compared with the value obtained from the sample of the shVec-transfected cells. (c) The tsp53-H1299 cells were transfected with a plasmid expressing wild-type (WT) or mutant NF-YA (m28 or m29) together with pGL3-Luc-PLK1 and pRL-TK. Cells were shifted to $32^{\circ} \mathrm{C}$ for the induction of functional p53, and luciferase activities were assayed at $24 \mathrm{~h}$ after transfection. (a-c) Bars represent the mean \pm S.D. from three independent experiments $\left({ }^{*} P<0.05 ;{ }^{* *} P<0.01 ;{ }^{* \star} P<0.001\right.$, Student's $t$-test)

with NF-YA or have a repressive effect on PLK1 transcription. Several possible scenarios to explain these results are as follows: (1) the exchange of NF-YA-binding partners might require a transient CDK2/p21/NF-YA trimeric complex formation. (2) The binding of p21 to CDK2 may induce a conformational change as a prerequisite for subsequent p21/NF-YA interaction on the PLK1 promoter. (3) The binding of p21 to CDK2 may result in the inhibition of CDK2 phosphorylation on NF-YA and the unphosphorylated NF-YA can then interact with p21. CDK2 was reported to activate NF-YA by phosphorylating its Ser-291 and Ser-297 residues. $^{22}$ It is conceivable that we could detect the p21$\mathrm{PRG/NF-YA}$ interaction in our in vitro binding assays because we used recombinant NF-YA from $E$. coli that had not been phosphorylated at the CDK2 substrate sites. Consistent with this scenario, our preliminary data showed that an NF-YA mutant, which is unable to be phosphorylated by CDK2 was more potent in supporting p21-dependent repression of PLK1 than the phosphomimetic mutant (unpublished results).

In the cellular response to the DNA damage stress, both p21-dependent and -independent mechanisms are used by p53 to ensure the suppression of PLK1. Our finding that p21 mediates the DNA damage-induced p53-dependent suppression of PLK1 does not exclude the possibility of direct suppression of PLK1 transcription by p53. The binding of p53 to a promoter element (p53RE2) 800-bp upstream of the CCAAT box is stimulated by DNA damage and responsible for inhibiting the PLK1 promoter activity independently of p21. ${ }^{13}$ In addition, p53 also represses a number of G2/M regulatory genes, such as $C D C 25 B, C D C 25 C, C C N B 1$ and $C D K 1{ }^{29-32}$ Promoters of these $\mathrm{G} 2 / \mathrm{M}$ genes share some regulatory elements with the PLK1 promoter, including the $\mathrm{CDE} / \mathrm{CHR}$ element and the CCAAT box. However, unlike the $P L K 1$ promoter that has one single CCAAT box, these G2/M gene promoters contain multiple CCAAT boxes immediately upstream of $\mathrm{CDE} / \mathrm{CHR} .{ }^{33}$ In regulating these $\mathrm{G} 2 / \mathrm{M}$ regulatory genes, p53 forms complexes with NF-YA and NF-YC bound on the CCAAT box promoter elements and converts NF-Y to a transcriptional repressor in the G2/M checkpoint response. ${ }^{33,34}$ In contrast, p53 is not detected in the CCAAT box region of the PLK1 promoter. ${ }^{13}$ Whether this difference in p53 recruitment is due to the presence of different numbers of the CCAAT box still requires investigation.

Our findings that the loss of NF-YA inhibits p53 activation and abolishes the p53-mediated protection from DNA damage-induced mitotic catastrophe have assigned a role to this ubiquitous transcription factor as a key molecule in the p53-dependent checkpoint functions. The NF-YA-dependent suppression of PLK1 is pivotal for maintaining the p53 checkpoint function. It has been reported that PLK1 inhibits p53 transcriptional activity via direct binding; thus, inhibition of PLK1 by NF-YA should relieve its suppression on the p53 activity. ${ }^{11,35}$ In this sense, NF-YA may function as an important component of the auto-regulatory loop that is required to maintain p53/p21-mediated G2/M checkpoint activities. It is worth noting that the inactivation of NF-YA has been shown to functionally activate p53 and increase apoptosis in the absence of DNA damage. ${ }^{36}$ Thus, consistent with the dual roles of NF-YA in regulating G2/M regulatory genes, it seems that depending on whether or not the cells are stressed, NF-YA can exert opposite effects in regulating p53 activities.

PLK1 overexpression has been reported in a variety of cancers and is tightly linked to poor prognosis. ${ }^{8}$ Selective inhibitors of PLK1 have been shown to suppress tumor growth in vivo and in vitro; ${ }^{37}$ hence, this mitotic kinase has recently emerged as a potential target for anticancer drug development. $^{38}$ By demonstrating that NF-YA is necessary for p21-mediated PLK1 suppression during the DNA damage response, this study provides important mechanistic insight into how PLK1 may be regulated, which should aid in the development of novel strategies targeting PLK1 for cancer therapy. 
a

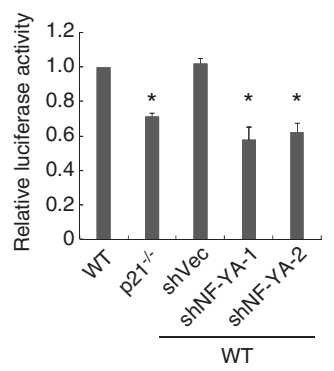

b
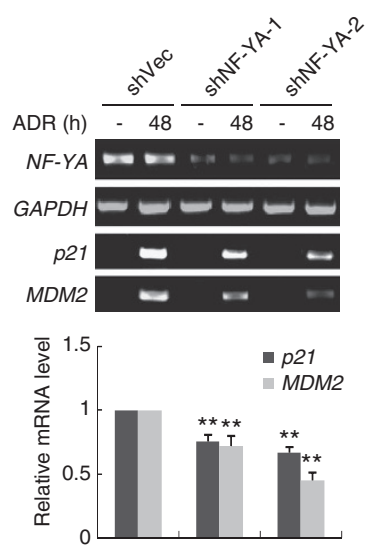

C

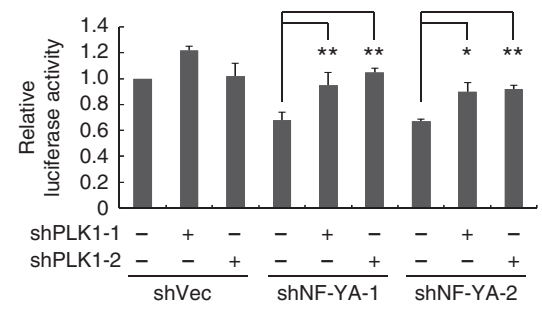

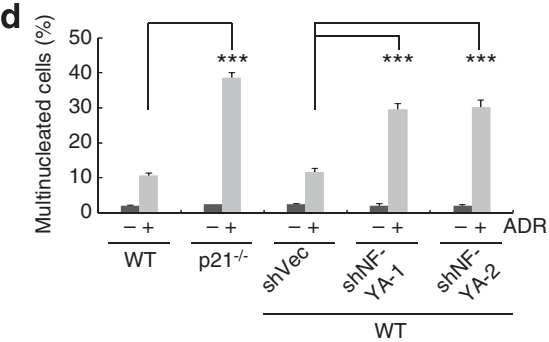

e

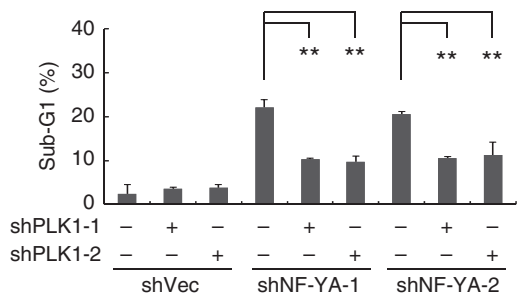

f
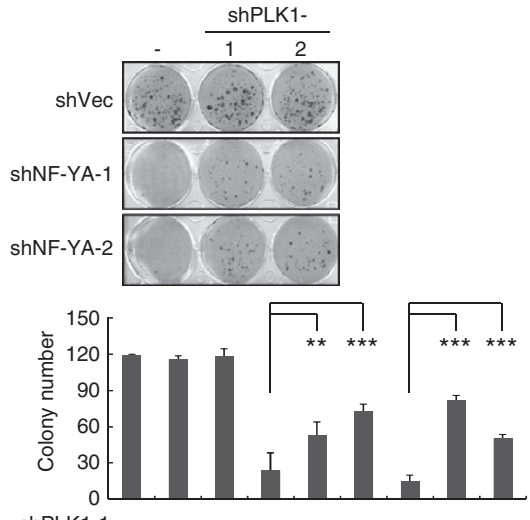

ShPLK1-1 - + - $-+-\quad+\quad+$

shPLK1-2 $\frac{--+}{\text { shVec }} \frac{--+}{\text { shNF-YA-1 }} \frac{--+}{\text { shNF-YA-2 }}$

Figure 6 NF-YA-mediated suppression of PLK1 expression is important for the p53 checkpoint function and prevention of mitotic death following DNA damage. (a) Wildtype (WT), p21 $1^{-1}$ or NF-YA knocked-down (shNF-YA-1 or shNF-YA-2) HCT116 cells were transfected with pRL-TK and pGUP.PA.8-p53RE (containing four copies of the p53 response element). Cells were treated with ADR for $12 \mathrm{~h}$ and subsequently cultured in the ADR-free medium for $24 \mathrm{~h}$, and analyzed for luciferase activities. Normalized luciferase activities were compared with that of the WT sample (which was set as 1). (b) The tsp53-H1299 cells were treated with ADR as in Figure 1c and subjected to RTPCR analysis for the mRNA levels of indicated genes. Target gene expression was normalized to GAPDH levels and compared with shVec samples. (c) The tsp53-H1299 cells transfected with the control (shVec) or shRNA-expressing plasmid(s) as indicated were co-transfected with pGUP.PA.8-p53RE and pRL-TK, treated with ADR, and subjected to luciferase assays as in Figure 1c. Normalized luciferase activities were compared with the value obtained from samples of shVec-control cells without $P L K 1 \mathrm{knockdown}$. (d) HCT116 cells were treated with ADR for $96 \mathrm{~h}$, stained with $4^{\prime}, 6^{\prime}$-diamidino-2-phenylindole (DAPI), and observed under the microscope to determine the percentage of multinucleated cells. (e) The tsp53-H1299 cells with or without PLK1 and/or NF-YA depletion were treated with ADR for $96 \mathrm{~h}$, and analyzed by flow cytometry for DNA content to determine the percentages of subG1 cells. (f) The tsp53-H1299 cells as in (e) were treated with ADR and cultured at $32^{\circ} \mathrm{C}$ for 6 days before being re-plated in 6-well plates. Cells were allowed to grow at $38^{\circ} \mathrm{C}$ for 10 more days, and colonies were scored after staining with crystal violet. Quantitative results from three experiments are shown. (a-f) Bars represent the mean \pm S.D. $\left({ }^{*} P<0.05 ;{ }^{* \star} P<0.01 ;{ }^{* * *} P<0.001\right.$, Student's $t$-test $)$

\section{Materials and Methods}

Cell culture. H1299 (p53-null human non-small cell lung carcinoma) cells were cultured at $37^{\circ} \mathrm{C}$ in RPMl- 1640 supplemented with $10 \%$ fetal bovine serum (FBS). The mutant tsp53 $3^{\mathrm{V} 143 \mathrm{~A}}-\mathrm{H} 1299$ and the control neo-H1299 cell lines were maintained in RPMI-1640 supplemented with $10 \%$ FBS; these clones were cultured at $38^{\circ} \mathrm{C}$ as p53-null cells, or transferred to $32^{\circ} \mathrm{C}$ for the induction of functional p53 in tsp53 $3^{\mathrm{V} 143 \mathrm{~A}}-\mathrm{H} 1299$ cells. Cells of p21-deficient tsp53-H1299 (p21-KD) and vector control clones were cultured at $38^{\circ} \mathrm{C}$ in the presence of $400 \mu \mathrm{g} / \mathrm{ml}$ hygromycin and $0.2 \mathrm{mg} / \mathrm{ml} \mathrm{G} 418$. 293T (human embryonic kidney cells with wild-type p53 alleles and residual p53 activities despite the presence of SV40 large T antigen), and HCT116 (human colorectal carcinoma cells with wild-type p53) and its isogenic (p53 $3^{-1-}$ or $\mathrm{p} 21^{-1-}$ ) cell lines (provided by Dr. Tzu-Hao Cheng at the Institute of Biochemistry and Molecular Biology, National Yang-Ming University, Taipei, Taiwan) were cultured at $37^{\circ} \mathrm{C}$ in DMEM supplemented with $10 \%$ FBS. All cells were maintained in a humidified atmosphere of $5 \%$ $\mathrm{CO}_{2} / 95 \%$ air.
Immunoblotting analysis. Cells were lysed in a lysis buffer $(20 \mathrm{mM}$ Tris- $\mathrm{HCl}, \mathrm{pH} 7.5,150 \mathrm{mM} \mathrm{NaCl}, 1 \mathrm{mM} \mathrm{Na} 2$ ethylenediaminetetraacetic acid (EDTA), $1 \mathrm{mM}$ EGTA, $1 \%$ Triton X-100 and a protease inhibitor cocktail added immediately before use) on ice for $30 \mathrm{~min}$ and centrifuged at $12000 \mathrm{~g}$ at $4{ }^{\circ} \mathrm{C}$ for $15 \mathrm{~min}$. Proteins (40- $\mu$ g samples) were subjected to $8-12 \%$ SDS-PAGE, transferred to the Hybond-C Extra membrane (Amersham Biosciences, Piscataway, NJ, USA), and incubated with antibodies against proteins of interest, including NF-YA (Santa Cruz Biotechnology, Santa Cruz, CA, USA), CDK2 (Santa Cruz Biotechnology), PLK1 (Zymed Laboratories, South San Francisco, CA, USA), p21 (Calbiochem, Merck KGaA, Darmstadt, Germany), p53 (Calbiochem), GAPDH (Abcam, Cambridge, UK) and $\alpha$-tubulin (Sigma-Aldrich, St Louis, MO, USA). Protein signals were detected using a chemiluminescence detection system (Amersham Biosciences) and captured on the Super RX X-ray film (FujiFilm, Tokyo, Japan).

Plasmid construction. The firefly luciferase reporter construct pGL3-LucPLK1 was generated by cloning the -1717 to +58 (numbered relative to the 


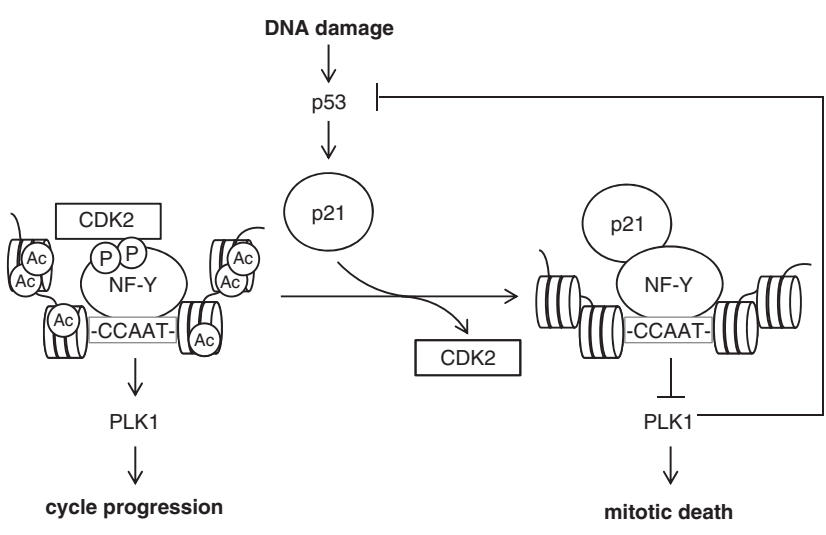

Figure 7 NF-YA participates in the transcriptional regulation of PLK1 expression. We propose a model in which the dynamic interplay among CDK2, p21 and NF-YA on the promoter fine tunes PLK1 expression. In unstressed cells, CDK2 associates with NF-YA bound on the CCAAT box in the PLK1 promoter to allow cell cycle progression. DNA damage activates p53 and induces p21 to replace CDK2 in binding to NF-YA, which causes $P L K 1$ repression and prevents mitotic cell death. In cells depleted of p21 or NF-YA, DNA damage fails to repress PLK1 expression; as a result, PLK1 acts to attenuate functions of p53, thereby resulting in mitotic catastrophe. Ac, acetylation of histones; P, phosphorylation of NF-YA

translational start site) PLK1 promoter fragment through polymerase chain reactions (PCRs) amplifications and subsequent subcloning of the PCR product into Kpnl/Bglll sites of the pGL3-Basic plasmid (Promega). Primers used were PLK1 promoter-F (5'-GGGGTACCCCGACTGTGGGAGGCTTACACCTGGTTTCCCT-3') and PLK1 promoter-R (5'-GAAGATCTTCCGCTCCTCCCCGAATTCAAACGC GGCGCTG-3'). Mutagenesis was performed using a QuikChange site-directed mutagenesis kit (Agilent Technologies, Santa Clara, CA, USA) according to the manufacturer's protocols to generate variant reporter constructs with specific mutations in the PLK1 promoter, or a p21 mutant (p21-PRG)-expressing construct. Specific mutagenic primer pairs were PLK1 mCCAAT-F (5'-GGATTTT AAATCCCCGCGGCAGCTCAGTGGCGCG-3') and PLK1 mCCAAT-R (5'-CC CGCCCAAACCTAAAATTTAGGGGCGCCG-3') for the mCCAAT reporter construct, and p21-PRG-F (5'-AGCGATGGAACTTCGACTTTCCGCGAGGACTG GAGGGTGACTTCG-3') and p21-PRG-R (5'-AAAGTCGAAGTCCATCGCT CACGGGCCTCCTGGAT-3') for the p21-PRG expression construct; underlined sequences shown in the primers are the mutated nucleotides. Multiple (12-16) PCR cycles were used to amplify the entire vector, and the PCR products were digested with $D p n l$ for $1 \mathrm{~h}$ at $37^{\circ} \mathrm{C}$ and subsequently transformed into E. coli. DNA was extracted from transformants and the target sequence was verified by DNA sequencing. For constructing GST fusion protein-expressing plasmids, NF-YA and p21 cDNA fragments were generated by PCR amplifications using specific primers. PCR fragments were ligated, in-frame, into pGEX-3X-KS (Amersham Biosciences) or pREST A (Invitrogen, Carlsbad, CA, USA) and transformed into BL21.

Luciferase reporter assay. Cells were co-transfected with a luciferase reporter plasmid, such as pGL3-Luc-PLK1, pGL3-Luc-mutPLK1 (with a mutated CCAAT box) or pGUP.PA.8-p53RE (a luciferase reporter construct containing four copies of p53RE), together with the Renilla luciferase control vector pRL-TK (Promega, Fitchburg, WI, USA). Lysates were prepared $24 \mathrm{~h}$ after transfection and assayed for the luciferase activity using a dual-luciferase reporter assay system (Promega). The activity of the firefly luciferase was normalized to that of the Renilla luciferase in the same assayed sample.

Lentiviral production and transduction. Plasmids for the expression of shRNAs targeting NF-YA (TRCN0000014298 and TRCN0000014930) or PLK1 (TRCN0000121073 and TRCN0000121325) were obtained from the National RNAi Core Facility Platform (located at the Institute of Molecular Biology/Genomic Research Center, Academia Sinica), which is supported by the National Core Facility Program for Biotechnology, Taiwan. Viral packaging was performed as described. ${ }^{11}$ For target cell transduction, cells were grown to $60 \%$ confluency, and the virus-containing media were added to cells with polybrene $(8 \mu \mathrm{g} / \mathrm{ml})$. At $24 \mathrm{~h}$ after transduction, the virus-containing media were removed and cells were collected for further experiments. As shNF-YA expression caused a rapid decrease in the number of HCT116 cells, it was not possible to select for infected cells; however, the efficacy of each batch of the NF-YA shRNA-expressing lentiviral particles was assessed by immunoblotting for NF-YA before conducting further experiments.

In vitro binding assay. E. coli transformants carrying plasmids for different fusion proteins were grown in the presence of $0.1 \mathrm{mM}$ isopropyl- $\beta$-D-thiogalactoside for $3.5 \mathrm{~h}$ at $30^{\circ} \mathrm{C}$ to induce the expression of fusion proteins. For purification of GST-tagged proteins, bacteria were lysed by sonication in buffer A $50 \mathrm{mM}$ Tris- $\mathrm{HCl}, \mathrm{pH} 7.4,50 \mathrm{mM} \mathrm{NaCl}, 5 \mathrm{mM}$ dithiothreitol, $1 \mathrm{mM}$ phenylmethylsulfonyl fluoride, $1 \%$ Triton X-100, $1 \mathrm{mM}$ EDTA and $15 \%$ glycerol) and the lysates were filtered through a $0.45-\mu \mathrm{m}$ filter membrane. GST-tagged proteins were purified using glutathione-sepharose beads in buffer A. For purifying His-tagged proteins, bacteria were lysed by sonication in the lysis buffer $(50 \mathrm{mM}$ Tris- $\mathrm{HCl}, \mathrm{pH} 8$, $300 \mathrm{mM} \mathrm{NaCl}, 20 \mathrm{mM}$ imidazole and $0.05 \%$ Tween 20 ) and the lysates were filtered through a $0.45-\mu \mathrm{m}$ filter membrane and subsequently incubated with the Ni-NTA resins (Qiagen, Hilden, Germany). His-tagged proteins bound on resins were eluted in a lysis buffer containing $200 \mathrm{mM}$ imidazole. The eluted proteins were concentrated and exchanged in phosphate-buffered saline (PBS) by Microcon YM-3 (Millipore, Billerica, MA, USA) and were stored at $-70^{\circ} \mathrm{C}$. The $\left[{ }^{35} \mathrm{~S}\right]$ Met-labeled proteins were prepared using the TNT Quick Coupled Transcription/Translation system (Promega) according to the manufacturer's instructions. For binding assays, the GST-tagged proteins were immobilized on glutathione-sepharose beads and incubated with purified His-tagged proteins at $4{ }^{\circ} \mathrm{C}$ overnight or with the $\left[{ }^{35} \mathrm{~S}\right]$ Met-labeled proteins at $4{ }^{\circ} \mathrm{C}$ for $4 \mathrm{~h}$. After washing with PBS, the beads-bound proteins were subjected to 10\% SDS-PAGE and immunoblotting or autoradiography.

RNA extraction and RT-PCR. Total RNA was isolated with the TRIzol reagent (Invitrogen). The RNA ( $5 \mu \mathrm{g}$ ) was reverse transcribed into $\mathrm{CDNA}$ using the oligo-d(T) $)_{12-18}$ primer and reverse transcriptase in a $20-\mu$ l reaction mixture. Amplification was performed for CDKN1A (20 cycles) using primers p21-F (5'-ATGTCAGAACCGGCTGGGGA-3') and p21-R (5'-TTAGGGCTTCCTCTTG GAGA-3'), for MDM2 (20 cycles) using primers MDM2-F (5'-CAATCCACAAAG TAAATAGCA-3') and MDM2-R (5'-CACAGAACATTAAACAGTACA-3'), for NFYA (35 cycles) using primers NF-YA-F (5'-CTGTGCCTGCTATCCAAAGAATCCC- $3^{\prime}$ ) and NF-YA-R (5'-ACACTCGGATGATCTGTGTCATTGC-3'), for PLK1 (30 cycles) using primers PLK1-F (5'-GCAGATCAACTTCTTCCAGGATC-3') and PLK1-R (5'-GCATAAAGCCAAGGAAAGGACAG-3') and for GAPDH (25 cycles) using primers GAPDH-F (5'-AAGTATGACAACAGCCTCAAGA- $\left.3^{\prime}\right)$ and GAPDH-R (5'-CACCACCTTCTTGATGTCATCA-3'). The thermocycling program used was: $30 \mathrm{~s}$ at $95^{\circ} \mathrm{C}, 30 \mathrm{~s}$ at $58{ }^{\circ} \mathrm{C}$ and $35 \mathrm{~s}$ at $72{ }^{\circ} \mathrm{C}$ in each cycle.

Co-immunoprecipitation. For immunoprecipitation, each 1-mg sample of cell lysates was pre-cleared by incubating with $20 \mu \mathrm{l}$ of protein A-sepharose beads (Amersham Biosciences) for $1 \mathrm{~h}$ at $4^{\circ} \mathrm{C}$ to reduce nonspecific binding, and subsequently allowed to react with $1 \mu \mathrm{g}$ of an antibody against the protein of interest for $16 \mathrm{~h}$ at $4^{\circ} \mathrm{C}$; the antibody was incubated with protein A-sepharose beads for $1 \mathrm{~h}$ at $4{ }^{\circ} \mathrm{C}$ beforehand. After washing, proteins in the immunoprecipitated complexes were separated by $10 \%$ SDS-PAGE and detected by using a chemiluminescence detection system (Amersham Biosciences) and captured on the Super RX X-ray films (Fuji Film).

Chromatin immunoprecipitation. The assay was performed as described previously. ${ }^{11}$ The antibodies used include NF-YA (Santa Cruz Biotechnology), CDK2 (Santa Cruz Biotechnology), p21 (Santa Cruz Biotechnology) and Flag (Sigma-Aldrich). Promoter regions of PLK1 (Figure 2a) and CDC25A were amplified by $P C R$. Primer pairs used were PLK1 region A-F $\left(5^{\prime}-A G\right.$ ACTAGGAGGTGGTGGTGTTGGCA-3') and PLK1 region A-R (5'-TGAAGGACAA TGAGGACAATGATGC-3'), PLK1 region B-F (5'-CCCTGGCCCCGAGGTAGAGG AAGATTTT-3') and PLK1 region B-R (5'-GAAGATCTTCCGCTCCTCCCCGAATTC AAACGCGGCGCTG- $3^{\prime}$ ), and CDC25A-F (5'-AGAGCCGATGACCTGGCAGAG TCCC-3') and CDC25A-R (5'-GTCTTCGCTGTTCTCCCACCCGCTT-3').

Fluorescence cell staining. Cells grown on glass coverslips were fixed in $5 \%$ formaldehyde for $15 \mathrm{~min}$ at $25^{\circ} \mathrm{C}$ and incubated in PBS containing $0.5 \%$ Triton $\mathrm{X}-100$ for $15 \mathrm{~min}$. Cells were subsequently stained with $4^{\prime}, 6^{\prime}$-diamidino-2phenylindole for $45 \mathrm{~min}$ in the dark and examined under a fluorescence microscope (ECLIPSE E800, Nikon, Tokyo, Japan). 
Flow cytometry. Cells $\left(1 \times 10^{6}\right)$ were fixed in ice-cold $70 \%$ ethanol at $4{ }^{\circ} \mathrm{C}$ for $16 \mathrm{~h}$. To perform cell cycle analysis, cells were treated with RNase A $(1 \mathrm{mg} / \mathrm{ml})$, stained with propidium iodide $(20 \mu \mathrm{g} / \mathrm{ml})$, and subsequently subjected to flow cytometry using a FACScan system (BD Biosciences, San Jose, CA, USA). Data were processed using the WinMDI software (http://facs.scripps.edu/software.html).

Clonogenic survival assay. Cells were exposed to ADR for 6 days, trypsinized and subsequently re-seeded in a six-well plate $\left(1.2 \times 10^{4}\right.$ cells per well) and cultured at $38^{\circ} \mathrm{C}$. After 10 days, the colonies were stained with crystal violet and scored.

\section{Conflict of Interest}

The authors declare no conflict of interest.

Acknowledgements. We thank Dr. Tzu-Hao Cheng for providing cell lines, and Min-Lun Li for critical reading of the manuscript. This work was supported by grants NSC98-2320-B-010-009-MY3 to F-FW and NSC99-2320-B-010-023-MY3 to T-YC from the National Science Council, DOH102-TD-C-111-007 to T-YC from the Department of Health, and 100AC-T506 to F-FW and 102AC-TC15 to M-YC from the Ministry of Education, Taiwan.

1. Bruinsma W, Raaijmakers JA, Medema RH. Switching Polo-like kinase-1 on and off in time and space. Trends Biochem Sci 2012; 37: 534-542.

2. Lens SM, Voest EE, Medema RH. Shared and separate functions of polo-like kinases and aurora kinases in cancer. Nat Rev Cancer 2010; 10: 825-841.

3. van Vugt MA, Medema RH. Getting in and out of mitosis with Polo-like kinase-1. Oncogene 2005; 24: 2844-2859.

4. Martin BT, Strebhardt K. Polo-like kinase 1: target and regulator of transcriptional control. Cell Cycle 2006; 5: 2881-2885.

5. Uchiumi T, Longo DL, Ferris DK. Cell cycle regulation of the human polo-like kinase (PLK) promoter. J Biol Chem 1997; 272: 9166-9174.

6. Lindon C, Pines J. Ordered proteolysis in anaphase inactivates Plk1 to contribute to proper mitotic exit in human cells. $J$ Cell Biol 2004; 164: 233-241.

7. Smits VA, Klompmaker R, Arnaud L, Rijksen G, Nigg EA, Medema RH. Polo-like kinase-1 is a target of the DNA damage checkpoint. Nat Cell Biol 2000; 2: 672-676.

8. Takaki T, Trenz K, Costanzo V, Petronczki M. Polo-like kinase 1 reaches beyond mitosis-cytokinesis, DNA damage response, and development. Curr Opin Cell Biol 2008; 20: $650-660$.

9. Yuan JH, Feng Y, Fisher RH, Maloid S, Longo DL, Ferris DK. Polo-like kinase 1 inactivation following mitotic DNA damaging treatments is independent of ataxia telangiectasia mutated kinase. Mol Cancer Res 2004; 2: 417-426.

10. van Vugt MA, Smits VA, Klompmaker R, Medema RH. Inhibition of Polo-like kinase-1 by DNA damage occurs in an ATM- or ATR-dependent fashion. J Biol Chem 2001; 276: $41656-41660$

11. Lin YC, Sun SH, Wang FF. Suppression of Polo like kinase 1 (PLK1) by p21(Waf1) mediates the p53-dependent prevention of caspase-independent mitotic death. Cell Signal 2011; 23: 1816-1823.

12. Jackson MW, Agarwal MK, Yang J, Bruss $P$, Uchiumi $T$, Agarwal ML et al. p130/p107/p105Rb-dependent transcriptional repression during DNA-damage-induced cell-cycle exit at G2. J Cell Sci 2005; 118(Pt 9): 1821-1832.

13. McKenzie L, King S, Marcar L, Nicol S, Dias SS, Schumm K et al. p53-dependent repression of polo-like kinase-1 (PLK1). Cell Cycle 2010; 9: 4200-4212.

14. Zhu H, Chang BD, Uchiumi T, Roninson IB. Identification of promoter elements responsible for transcriptional inhibition of polo-like kinase 1 and topoisomerase llalpha genes by p21(WAF1/CIP1/SDI1). Cell Cycle 2002; 1: 59-66.

15. Brauninger A, Strebhardt $\mathrm{K}$, Rubsamen-Waigmann $\mathrm{H}$. Identification and functional characterization of the human and murine polo-like kinase (PIk) promoter. Oncogene 1995; 11: $1793-1800$.

16. Maity SN, de Crombrugghe B. Role of the CCAAT-binding protein CBF/NF-Y in transcription. Trends Biochem Sci 1998; 23: 174-178.
17. Mantovani R. The molecular biology of the CCAAT-binding factor NF-Y. Gene 1999; 239 : $15-27$.

18. Ceribelli M, Dolfini D, Merico D, Gatta R, Vigano AM, Pavesi $G$ et al. The histone-like NF-Y is a bifunctional transcription factor. Mol Cell Biol 2008; 28: 2047-2058.

19. Peng $Y$, Jahroudi $N$. The NFY transcription factor functions as a repressor and activator of the von Willebrand factor promoter. Blood 2002; 99: 2408-2417.

20. Peng Y, Stewart D, Li W, Hawkins M, Kulak S, Ballermann B et al. Irradiation modulates association of NF-Y with histone-modifying cofactors PCAF and HDAC. Oncogene 2007; 26: $7576-7583$

21. Zhu X, Wang Y, Pi W, Liu H, Wickrema A, Tuan D. NF-Y recruits both transcription activator and repressor to modulate tissue- and developmental stage-specific expression of human gamma-globin gene. PloS One 2012; 7: e47175.

22. Chae HD, Yun J, Bang YJ, Shin DY. Cdk2-dependent phosphorylation of the NF-Y transcription factor is essential for the expression of the cell cycle-regulatory genes and cell cycle G1/S and G2/M transitions. Oncogene 2004; 23: 4084-4088.

23. Yun J, Chae HD, Choi TS, Kim EH, Bang YJ, Chung J et al. Cdk2-dependent phosphorylation of the NF-Y transcription factor and its involvement in the p53-p21 signaling pathway. J Biol Chem 2003; 278: 36966-36972.

24. Vigneron A, Cherier J, Barre B, Gamelin E, Coqueret $\mathrm{O}$. The cell cycle inhibitor p21waf1 binds to the myc and cdc25A promoters upon DNA damage and induces transcriptional repression. J Biol Chem 2006; 281: 34742-34750.

25. Xiong Y, Hannon GJ, Zhang H, Casso D, Kobayashi R, Beach D. p21 is a universal inhibitor of cyclin kinases. Nature 1993; 366: 701-704.

26. Garcia-Wilson E, Perkins ND. p21WAF1/CIP1 regulates the $\mathrm{p} 300$ sumoylation motif CRD1 through a C-terminal domain independently of cyclin/CDK binding. Cell Cycle 2005; 4: 1113-1119.

27. Mantovani R, Li XY, Pessara U, Hooft van Huisjduijnen R, Benoist C, Mathis D. Dominant negative analogs of NF-YA. J Biol Chem 1994; 269: 20340-20346.

28. Lin YC, Wang FF. Mechanisms underlying the pro-survival pathway of $p 53$ in suppressing mitotic death induced by adriamycin. Cell Signal 2008; 20: 258-267.

29. Dalvai M, Mondesert O, Bourdon JC, Ducommun B, Dozier C. Cdc25B is negatively regulated by p53 through Sp1 and NF-Y transcription factors. Oncogene 2011; 30: 2282-2288.

30. St Clair S, Giono L, Varmeh-Ziaie S, Resnick-Silverman L, Liu WJ, Padi A et al. DNA damage-induced downregulation of $\mathrm{Cdc} 25 \mathrm{C}$ is mediated by $\mathrm{p} 53$ via two independent mechanisms: one involves direct binding to the cdc25C promoter. Mol Cell 2004; 16: 725-736.

31. Innocente SA, Lee JM. p53 is a NF-Y- and p21-independent, Sp1-dependent repressor of cyclin B1 transcription. FEBS Lett 2005; 579: 1001-1007.

32. Yun J, Chae HD, Choy HE, Chung J, Yoo HS, Han MH et al. p53 negatively regulates cdc2 transcription via the CCAAT-binding NF-Y transcription factor. J Biol Chem 1999; 274: 29677-29682.

33. Imbriano C, Gurtner A, Cocchiarella F, Di Agostino S, Basile V, Gostissa M et al. Direct p53 transcriptional repression: in vivo analysis of CCAAT-containing G2/M promoters. Mol Cell Biol 2005; 25: 3737-3751.

34. Imbriano C, Gnesutta N, Mantovani R. The NF-Y/p53 liaison: well beyond repression. Biochim Biophys Acta 2012; 1825: 131-139.

35. Ando K, Ozaki T, Yamamoto H, Furuya K, Hosoda M, Hayashi S et al. Polo-like kinase 1 (PIk1) inhibits p53 function by physical interaction and phosphorylation. J Biol Chem 2004; 279: 25549-25561.

36. Gurtner A, Fuschi P, Martelli F, Manni I, Artuso S, Simonte G et al. Transcription factor NF-Y induces apoptosis in cells expressing wild-type p53 through E2F1 upregulation and p53 activation. Cancer Res 2010; 70: 9711-9720.

37. Schoffski P. Polo-like kinase (PLK) inhibitors in preclinical and early clinical development in oncology. Oncologist 2009; 14: 559-570.

38. Strebhardt K, Ullrich A. Targeting polo-like kinase 1 for cancer therapy. Nat Rev Cancer 2006; 6: 321-330

Ce) (i) $\odot$ Cell Death and Disease is an open-access journal
published by Nature Publishing Group. This work is
licensed under a Creative Commons Attribution-NonCommercial-
NoDerivs 3.0 Unported License. To view a copy of this license, visit
http://creativecommons.org/licenses/by-nc-nd/3.0/ 